\title{
Transmission of Digital Audio with Visible Light
}

\author{
Sergio Sandoval-Reyes, Arturo Hernandez-Balderas \\ CIC, Instituto Politécnico Nacional, CDMX, \\ Mexico \\ sersand@cic.ipn.mx, heba920908@gmail.com
}

\begin{abstract}
Communication by visual light or VLC by its acronym in English (Visual Light Communication), uses visible light from fluorescent lamps, or light from light emitting diode (LED) to transmit information. The transmission of information is done by modulating the intensity of the LED light. In the receiver the information is initially recovered through a photo-detector, which is usually connected to a device for the final recovering of the information. In this article we describe an application based on VLC, to transmit audio stored in a micro SD card using a shield mounted on an Arduino microcontroller, and several modules (called bits) of LittleBits. The use of modular components that allow simple interconnection, facilitated the development of this application.
\end{abstract}

Keywords: visual light communications, audio, Arduino.

\section{Introduction}

Visible Light Communication (i.e. VLC). [1, 2], transmits data by intensity modulation. It uses Light Emitter Diodes (LEDs) and light detectors at transmit and receive ends respectively. It works in the $380 \mathrm{~nm}$ to $780 \mathrm{~nm}$ optical band which is visible light and hence the name VLC $[3,4,5]$.

VLC can be used as a communications medium to transmit data by visible light for ubiquitous computing, because light-producing devices (such as indoor/outdoor lamps, traffic signs, commercial displays and car headlight and taillights) are used everywhere [6].

This paper describes a VLC application to transmit and receive digital audio using an Arduino microcontroller connected to a LED, and a light sensor connected to a speaker.

The remainder of this paper is organized as follows: Section 2 presents a summary of works related to the transmission of digital audio using VLC. Section 3 describes the design of our VLC application. Section 4 shows the results of the implementation. Finally, our conclusions and future work are presented in Section 5.

\section{Related Work}

Several research works on VLC technologies have been proposed. The most important are described in the following.

pp. 61-68; rec. 2017-09-08; acc. 2017-10-25 $61 \quad$ Research in Computing Science 138 (2017) 


\subsection{How LED based VLC Systems Works}

Typical LED based VLC systems are implemented using an intensity modulation and direct detection (IM/DD) scheme with a line-of-sight (LOS) configuration [7]. In the transmitter, IM is implemented through the modulation of the transmitted signal into the instantaneous optical power of the LED by controlling the radiant intensity with the forward current through the LED (High modulation frequencies are used to avoid flicker). In the receiver, the transmitted signal is recovered using direct detection (DD). In this simple method, a photodiode is used to convert the incident optical signal power into a proportional current. Figure 1 shows a general VLC link structure for an IM/DD based VLC system [8].

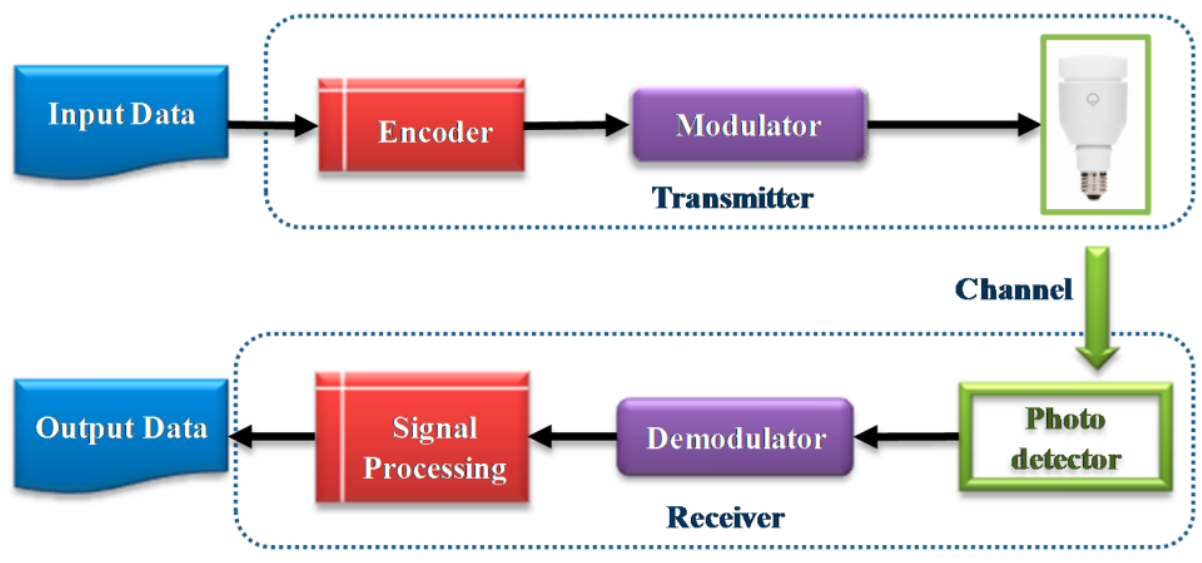

Fig. 1. A general VLC link structure.

\subsection{VLC Transmitter}

A typical LED based VLC transmitter contains a signal generator (information source), and a modulator, followed by the LED driver and the LED optics, see Figure 1 [9]. The modulation methods available for VLC, particularly for indoor applications, must support dimming and provide flicker mitigation. The modulating signals are used to switch LEDs at desired frequencies using LED drivers. These drivers rely on transconductance amplifiers to convert voltage signals to corresponding current signals to excite the light sources (LEDs) for both communication and illumination purposes.

\section{VLC Modulation}

Although there are different modulation schemes for VLC, mainly, on-off keying $(\mathrm{OOK})$, variable pulse-position modulation (VPPM), color shift keying (CSK) and orthogonal frequency division multiplexing (OFDM) [10], OOK is the most popular. OOK (On-Off-Keying) is the most commonly used IM/DD modulation scheme in VLC due to its simple implementation. In this method basically the LED intensity is 
changed between two distinguishable levels corresponding to the data bits ( 1 or 0$)$. See Figure 2. A modified OOK, called Variable OOK (VOOK) can provide dimming. It is achieved by changing the data duty cycle through pulse-width modulation (PWM), with only 1 bit of information carried per symbol period

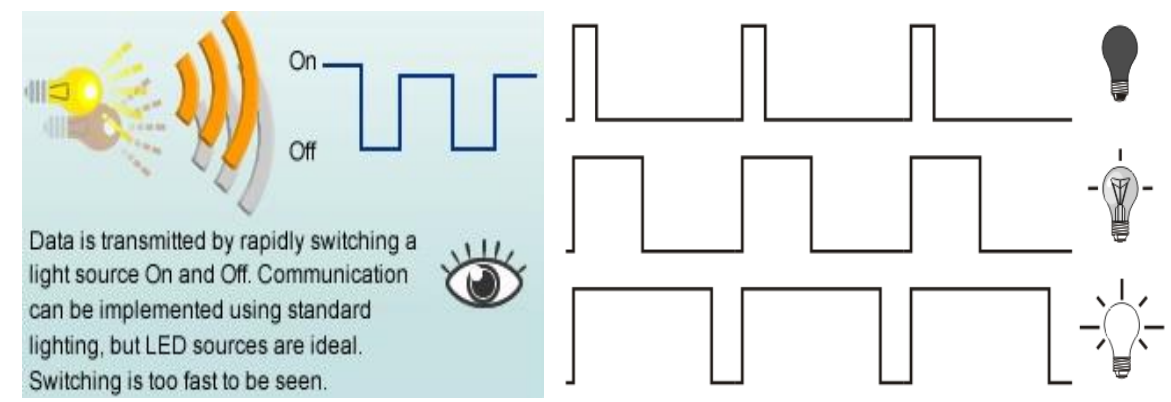

Fig. 2. On-Off Keying with PWM.

\subsection{VLC Receiver}

A typical optical receiver's front end consists of a photo detector followed by an amplifier. The photo detector can be a photo diode or a Light Detect Resistor (LDR). In both cases its impedance decreses in the presence of light and increses in its absense. See Figure 3. A simple photo detector can be used as a receiver since VLC exhibits no Doppler shift, and therefore it is not necessary a sophisticated receiver tracking algorithm.

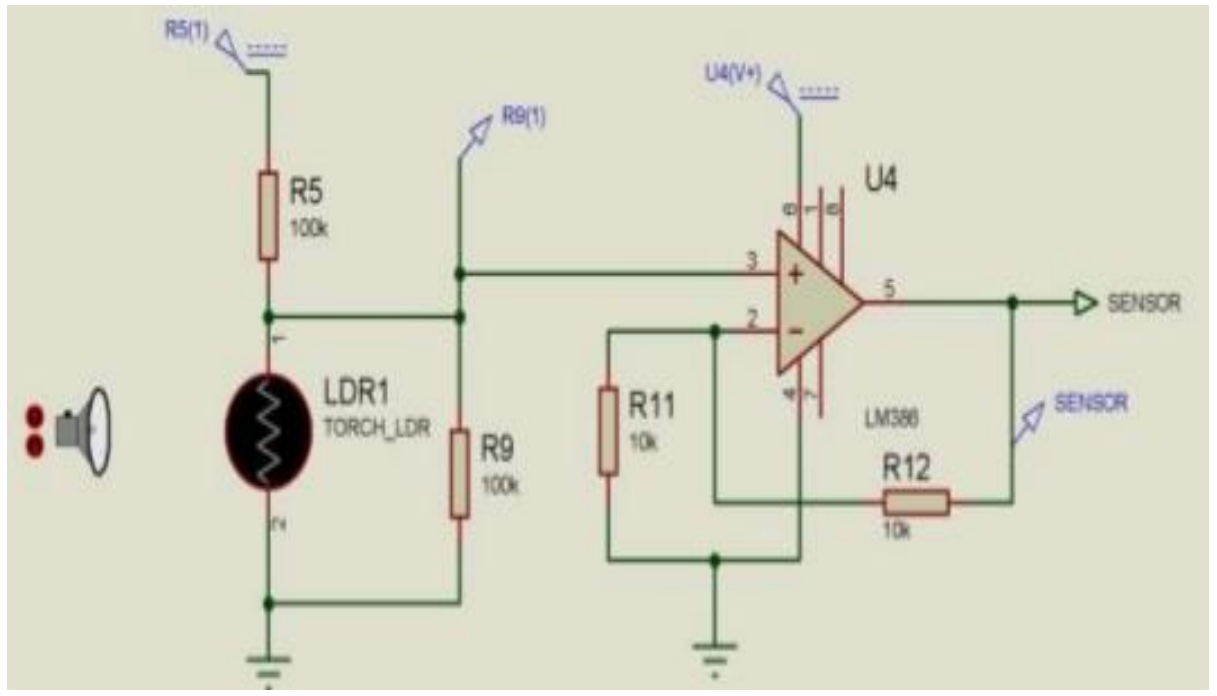

Fig. 3. A LDR photo detector followed by an amplifier. 


\section{Audio Streaming Using VLC}

In the following we develop a VLC application to transmit and receive realtime audio using light from a LED in the transmitter, and a photo detector in the receiver.

In order to do that, we will use in the transmitter as a data source, an Arduino microcontroller with a SD shield 3.0 [11] containing digital music in WAV format, and two LittleBits modules [12]: A microphone/amplifier bit and a bright LED bit. See Figure 4. The Arduino output ports produce OOK-PWM output signals, whose duty cicles represent the audio intensity.

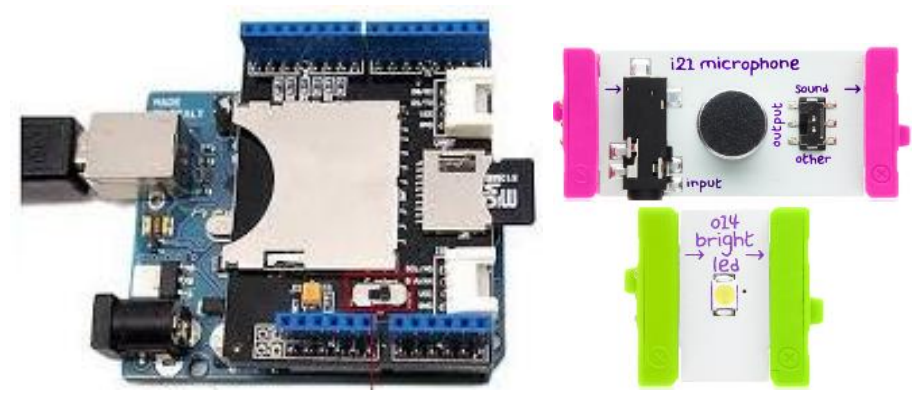

Fig. 4. An Arduino microcontroller with a SD Shield and two LittleBits modules.

While as in the receiver we will use two LittleBits components: A light sensor bit, and a speaker bit. See Figure 5.
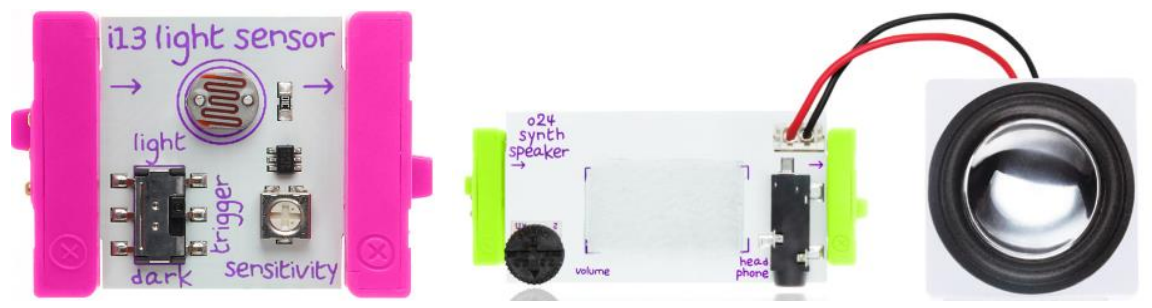

Fig. 5. Receiver components: Light sensor and speaker.

\subsection{Transmitting Digital Audio via VLC}

The Arduino (Master device) loads the WAV file from the micro-SD card (Slave device) in .wav format throught out pins d4 (CS: Chip Select), d11 (MOSI: Master Output Slave Input), d12 (MISO: Master Input Slave Output), and d13 (SCK: Signal Clock). It then generates an On-Off PWM signal and outputs it (pin 9), toward the microphone/amplifier component, which amplifies the On-Off PWM signal and feeds it to the bright LED bit component. The .wav file stored in the SD card has the following specifications: 8 bits per sample; $22050 \mathrm{~Hz}$ sampling rate; mono channel; and PCM unsigned 8-bit format. The Arduino code to execute the above mentioned is shown in Figure 6. 


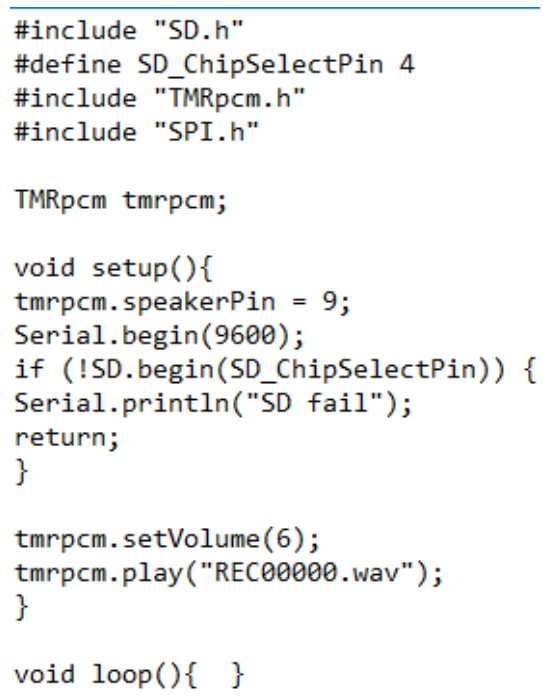

Fig. 6. Arduino code to play the audio .wav file stored in the SD card.

In this code (modified from [13]), the library "SD.h" allows Arduino-SD card communication; "TMRpcm" allows asynchronous playback of PCM/WAV files directly from the SD card; SPI (Serial Peripheral Interface) allows the transfer of audio data through the MISO, MOSI and SCK signals between the Arduino and the SD card.

\subsection{Receiving Digital Audio via VLC}

As it was mentioned, the streaming music sent as a LED light is received through a light sensor module which then sends it to the speaker module. This speaker module no only filters the audio signal but also amplify it. Using these LittleBits components makes these tasks very simple. See Figure 7.

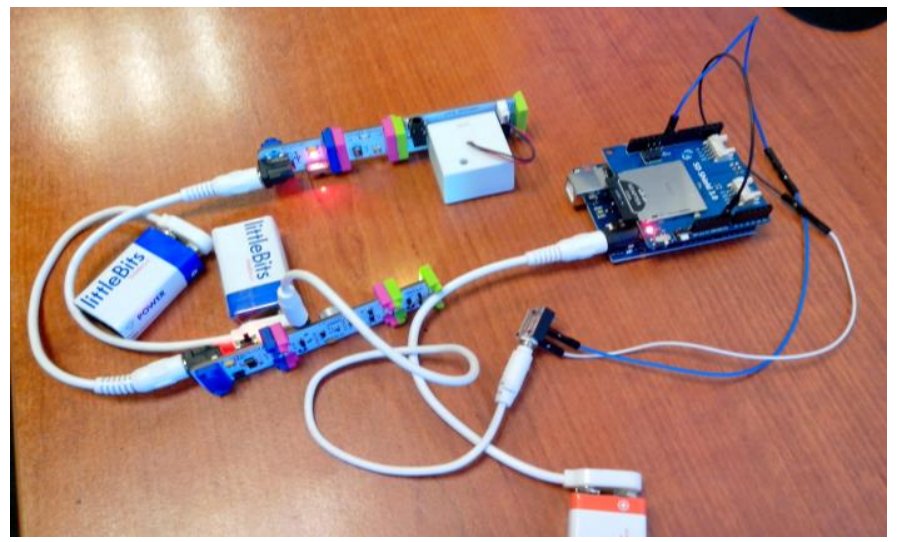

Fig. 7. Receiving digital audio via VLC. 


\section{$4 \quad$ Experiments and Results}

For the experiments we use as was mentioned an Arduino microcontroller with a SD card and several LittleBits components. The whole setup is shown in Figure 8.

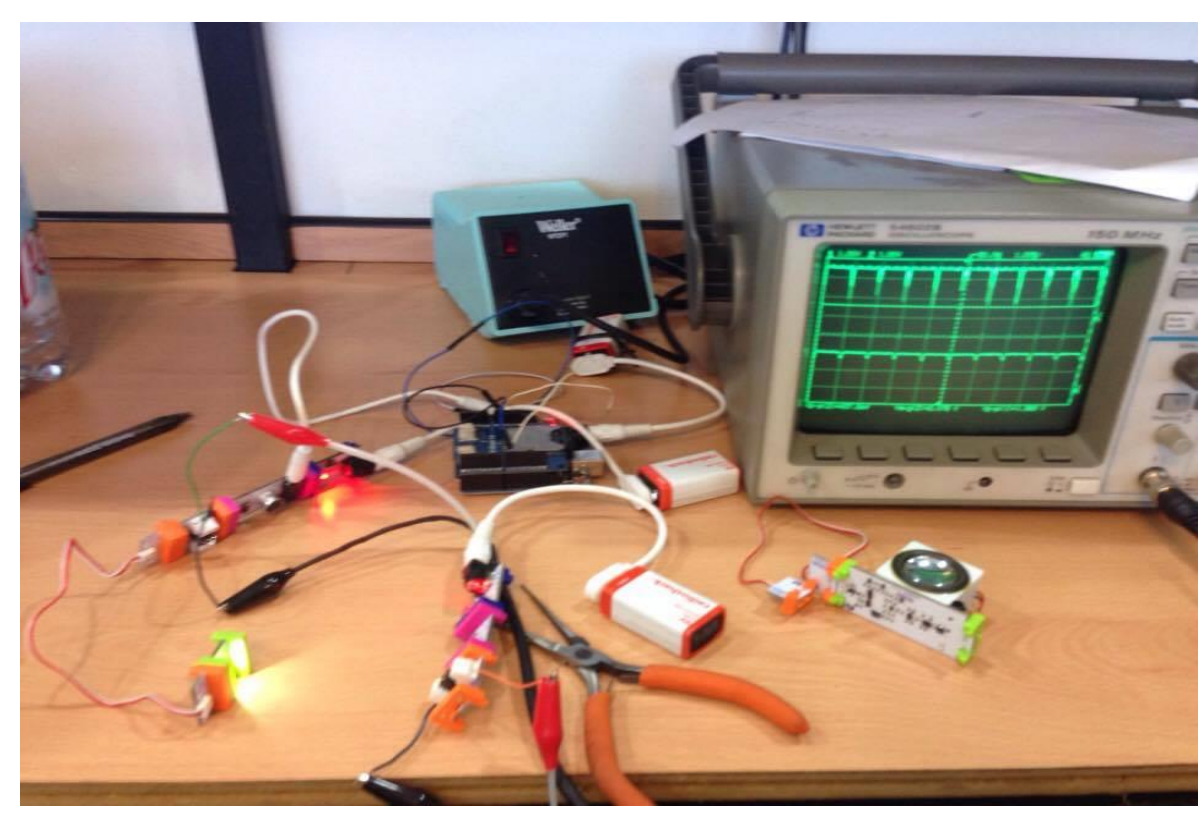

Fig. 8. Setup for the transmission and reception of digital audio using VLC.

Figure 8 shows the Arduino microcontroller with a SD shield. The shield contains a micro SD card which in turn contains the .wav file. The output from the Arduino is sent to the Mic-Amp-LED modules through an audio connector. Note that the audio connector has only two wires (digital audio signal and ground), because the audio has just one channel. Note also that both the Arduino and the LittleBits modules are energized with $9 \mathrm{~V}$ batteries.

This Figure 8 also shows the light coming from the LED and in the lower part of this figure, the backward face of the light sensor and the speaker modules. Naturally if an object blocks the light from the LED, the light sensor ceases of receiving this light and the music reproduced by the speaker, is interrupted.

\subsection{Discussion of Results}

The setup for the transmission and reception of audio using VLC worked well, although with a little noise. Naturally, this noise increases when the distance between the LED and the light sensor is greater. Also the brightness of the LED and the alignment between LED and light sensor also influence the performance. 
Using an oscilloscope we could capture the transmission signal feeding to the bright LED, thus as the reception signal recovered from the light sensor. This is shown in Figure 9. It can be seen that the light sensor signal is well recovered at $3 \mathrm{~cm}$.

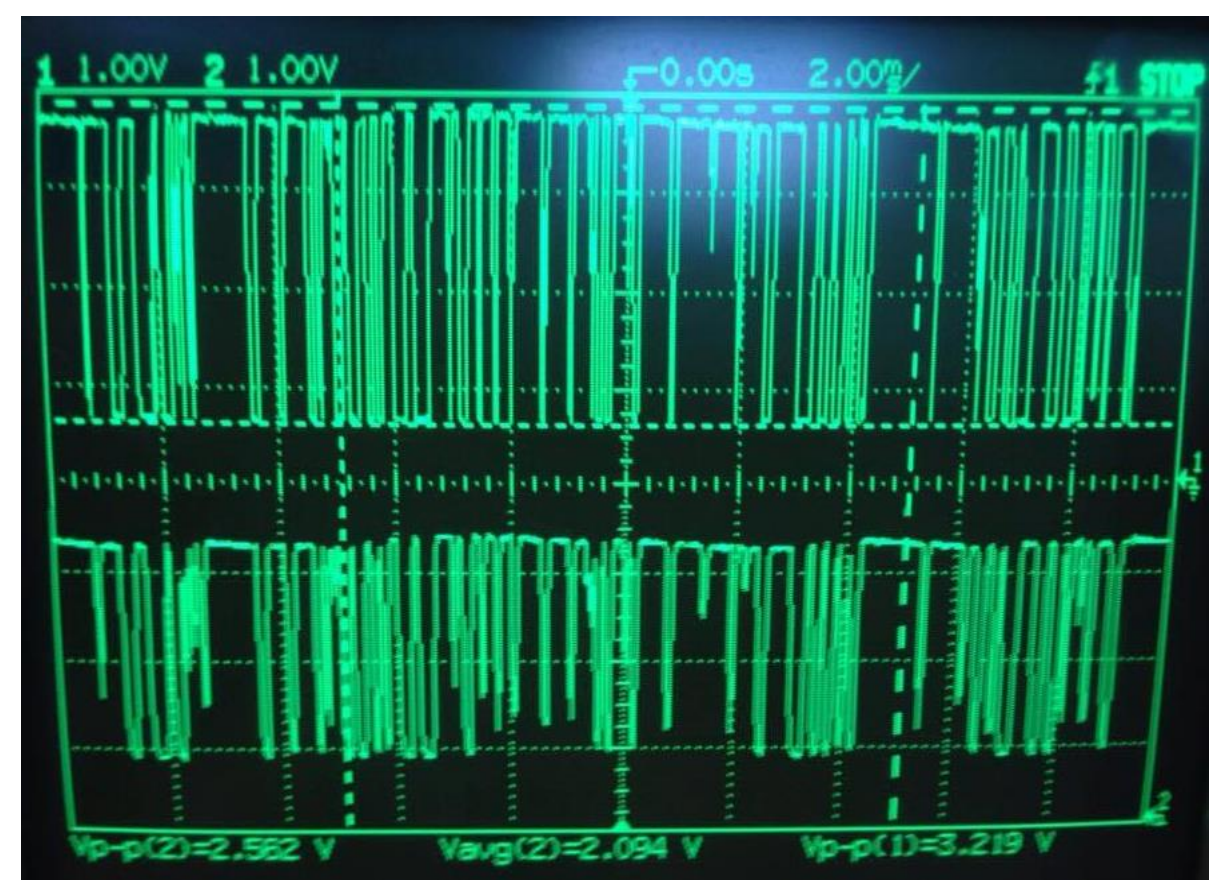

Fig. 9. Up: Digital transmission signal feeding the LED; Down: Received signal from the light sensor.

This work differs with respect to similar works as in [13], [14] and [15], in the following. In [13] it is required to use a specific microcontroller, in this case an "Arduino Due", which has a digital-to-analog converter at the output, to convert the audio in digital format to analog. In this work, the Arduino Due is not used, but the Arduino ONE which is more common. In [14], it is required to use two microcontrollers (one for the transmission and one for the reception), and the detection is done by biasing inversely to a LED diode (which modifies its resistance depending on the light received). In [15] an Arduino microcontroller is used for the transmission and a LDR photo sensor for reception, however no code is reported.

\section{Conclusions}

An audio transmission and reception application using VLC was developed using Arduino and LittleBits components. The application worked acceptably well although with a small presence of noise. It should be noted that this noise is also due to the fact that the wav file was recorded with only 8 bits per sample. In other words, it's not HiFi. 
Also, the performance of the application depends on the brightness of the emitter LED and the sensitivity of the photo sensor with respect to the light received from the LED and the surrounding light (in Figure 5 it can be seen that the light sensor has a control for graduating the sensitivity in the presence of high or low light). Additionally, the alignment between the LED and the light sensor influences the reception, and consequently the quality of the audio reproduction. This work could be improved by: 1) Increasing the emission power of the LED to increase the reception distance; and 2) Include video transmission by adding another LED light source to encode video.

Acknowledgements. This work was supported by the Instituto Politecnico Nacional; Project SIP 20171260.

\section{References}

1. Haas, H.: Wireless data from every light bulb. TED Ideas worth spreading, http://www.ted.com/talks/harald_haas_wireless_data_from_every_light_bulb (2011)

2. Tsonev, D., Videv, S. Haas, H.: Light fidelity (Li-Fi): towards all-optical networking. In: Proc. SPIE (Broadband Access Communication Technologies VIII) 9007(2) (2013)

3. Sherman, J.: How LED Light Bulbs could replace Wi-Fi. Digital Trends. http://www.digitaltrends.com/mobile/light-bulb-li-fi-wireless-internet/, last accessed 2016/05/01

4. Haas, H.: High-speed wireless networking using visible light. SPIE Newsroom (2013)

5. Vincent, J.: Li-Fi revolution: internet connections using light bulbs are 250 times faster than broadband. http://www.independent.co.uk/news/science/li-fi-revolution-internetconnections-using-light-bulbs-are-250-times-faster-than-broadband-8909320.html (2013) (last accessed on March 2016)

6. Jovicic, A., Li, J., Richardson, T.: Visible light communication: opportunities, challenges and the path to market. IEEE Communications Magazine, 51(12), 26-32 (2013)

7. Kamsula, P.: Desing and implementation of a bi-directional visible light communication testbed. University of Oulu, Department of Electrical and Information Engineering, Master's Thesis, 59 p. (2015)

8. He, Y., Ding, L., Gong, Y., Wang, Y.: Real-time audio \& video transmission system based on visible light communication. Optics and Photonics Journal, 3(02), 153 (2013)

9. Lee, K., Park, H.: Modulations for visible light communications with dimming control. IEEE photonics technology letters, 23(16), 1136-1138 (2011)

10. Arduino Homepage, https://www.arduino.cc/, last accessed 2017/09/01

11. LittleBIts Homepage, https://littlebits.cc/ last accessed 2017/09/01

12. Banzi, M., Fitzgerald, S., Guadalupi, A.: Simple Audio Player. (2015)

13. Jpiat, J.: Arduino Simple Visible Light Communication. https://github. com/jpiat/arduino/wiki/Arduino-Simple-Visible-Light-Communication, last accessed 2017/09/01

14. Yıldırım, G., Özen, Ö., Yüksel, H., İnci, M.: A Low-Cost Li-Fi Communication Setup. (2017) 